\title{
Sobrepeso na Adolescência de Filhos de Mães que Tiveram Distúrbios Glicêmicos na Gestação
}

\begin{abstract}
RESUMO
Objetivo: Observar se diferentes graus de hiperglicemia durante a gestação determinam diferentes freqüências de obesidade e suas comorbidades na adolescência dos filhos. Métodos: Participaram 73 filhos distribuídos em três grupos, segundo a tolerância à glicose materna: $\mathrm{G} 1(n=27)$ teste oral de tolerância à glicose (TOTG) e glicemia diária (GD) normais; G2 ( $n=23$ ) TOTG normal e GD elevada; G3 ( $n=23$ ) TOTG e GD alterados (diabetes melito gestacional-DMG). Todos foram submetidos à avaliação clínica (antropometria), a um questionário (dados neonatais, hábitos alimentares) e a dosagem basal de glicose e perfil lipídico sérico. A comparação entre os grupos foi feita por análise de variância e teste de Goodman. Resultados: As mães G3 apresentaram glicemia de jejum (GJ) e GD mais elevadas que as G2 e G1 (GJ: $98 \pm 10$ versus $83 \pm 5$ versus $78 \pm 10 \mathrm{mg} / \mathrm{dL} ; \mathrm{GD}: 104 \pm 12$ versus $93 \pm 7$ versus $85 \pm 9$ $\mathrm{mg} / \mathrm{dL}$, respectivamente; $\mathrm{p}<0,001)$. As mães $\mathrm{G} 2$ apresentaram $\mathrm{GD}$ mais elevada que as $\mathrm{G} 1(93 \pm 7$ versus $85 \pm 9 \mathrm{mg} / \mathrm{dL} ; \mathrm{p}<0,001)$. O peso de nascimento (PN) dos filhos G3 foi mais elevado que o dos G2 e G1 (3.667 \pm 527 versus $3.167 \pm 565$ e $3.282 \pm 401 \mathrm{~g}$, respectivamente; $p<0,05)$. Os filhos $\mathrm{G} 3$ apresentaram maior freqüência de sobrepeso que os $\mathrm{G} 1(52,2$ versus $14,8 \% ; p<0,05)$. Conclusões: Mães com DMG, apresentando GJ e GD elevadas, têm filhos com maior PN e maior freqüência de sobrepeso na adolescência. Esses filhos precisam ser acompanhados desde a infância. (Arq Bras Endocrinol Metab 2008;52/1:85-92)
\end{abstract}

Descritores: Diabetes melito gestacional; Sobrepeso; Adolescência; Hiperglicemia gestacional

\begin{abstract}
Overweight in Adolescent Offspring of Women with Hyperglycemia During Pregnancy.

AIM: To verify whether different hyperglycemia levels during pregnancy cause frequency differences in adolescent obesity and its morbidities in the offspring. Methods: Seventy-three children were divided into three groups according to maternal glucose tolerance: $\mathrm{G} 1(\mathrm{n}=27)$ normal oral glucose tolerance test (OGTT) and daily glycemia (DG); G2 $(n=23)$ normal OGTT and high DG; G3 $(n=23)$ abnormal OGTT and DG (gestational diabetes mellitus; GDM). All underwent clinical evaluation (anthropometry) a questionnaire(neonatal data, eating habits), and determination of fasting serum glucose and lipid profile measurement. Analysis of variance (ANOVA) and the Goodman's test were used to compare the groups. Results: G3 mothers showed higher fasting plasma glucose(FPG) and DG than G2 and G1(FPG: $93 \pm 10$ vs $83 \pm 5$ vs $78 \pm 10 \mathrm{mg} / \mathrm{dL}$; DG: $104 \pm 12$ vs $93 \pm 7$ vs $85 \pm 9 \mathrm{mg} / \mathrm{dL}$, respectively; $\mathrm{P}<0.001)$. G2 mothers had higher DG than $\mathrm{G} 1(93 \pm 7$ vs $85 \pm 9 \mathrm{mg} / \mathrm{dL} ; \mathrm{P}<0.001)$. G3 offspring birthweight was higher than $\mathrm{G} 1$ and $\mathrm{G} 2(3,667 \pm 527$ vs $3,167 \pm 565$ and $3,282 \pm 401 \mathrm{~g}$, respectively; $\mathrm{P}<0.05)$. More G3 offspring were overweight than $\mathrm{G} 1$ (52.2 vs $14.8 \%$;
\end{abstract}

artigo original

\author{
Elizabeth Fernandes Buzinaro \\ Carolina Bragiola BerchierI \\ Adriana lúcia Mendes Haddad \\ Carlos Roberto Padovani \\ Walkyria de Paula Pimenta
}

Departamento de Clínica

Médica, Faculdade de Medicina

de Botucatu, Universidade

Estadual Paulista (Unesp),

Botucatu, SP, Brasil (EFB, CBB,

ALMH, WPP); Departamento de Bioestatística, Instituto de Biociências de Botucatu, Unesp, Botucatu, SP, Brasil (CRP).

Recebido em 17/05/2007

Aceito em 09/1 1/2007 
$\mathrm{P}<0.05)$. Conclusions: Offspring of GDM mothers with fasting and daily hyperglycemia have higher birthweight and overweight frequency in adolescence. These children must be followed up from infancy. (Arq Bras Endocrinol Metab 2008;52/1:85-92)

Keywords: Gestational diabetes mellitus; Overweight; Adolescence; Gestational hyperglycemia

\section{INTRODUÇÃO}

$\mathrm{E}$ M TODO O MUNDO TEM AUMENTADO de forma alarmante a prevalência de obesidade e suas comorbidades na infância e na adolescência (1,2). Também têm sido diagnosticadas com elevada freqüência a síndrome metabólica e as doenças cardiovasculares nestas faixas etárias iniciais (1). Além desses dados, é conhecido que a maioria dos adolescentes obesos tornar-se-ão adultos obesos com todas suas complicações clínicas e sociais $(1,2)$. Assim, vários países já iniciaram medidas de combate ou mesmo prevenção da obesidade na infância e na adolescência $(1,2)$. Uma maneira efetiva de prevenção pode ser a identificação e a caracterização dos grupos de alto risco.

Em grupos populacionais tão diversos como aqueles predominantemente caucasianos e dos índios Pima observou-se maior freqüência de obesidade (3-5) e de diabetes melito tipo 2 (DMT2) $(4,5)$ na infância e na adolescência dos filhos de mães diabéticas ou com diabetes melito gestacional (DMG). As alterações metabólicas e hormonais maternas determinam morbidades futuras na prole por mecanismos propostos por Freinkel (6).

O peso de nascimento está muito relacionado com o risco futuro de desenvolvimento de obesidade $(7,8)$ e DMT2 $(7,9)$, uma vez que é determinado pelo meio intra-uterino, além dos fatores genéticos. Na maioria das populações, tanto os recém-nascidos classificados como grandes quanto os pequenos para a idade gestacional (GIG e PIG, respectivamente) têm risco maior de desenvolver ambas as morbidades, cujo mecanismo seria o da resistência à insulina.

Tallarigo e cols. (10) observaram que na $28 \pm 2^{\text {a }}$ semanas de gestação a glicemia de duas horas após uma carga oral de glicose (100 g), embora na faixa de normalidade, associou-se positivamente com a freqüência de macrossomia fetal. Também, em estudo mais detalhado do metabolismo da glicose materno, Farmer e cols.
(11) utilizaram o teste endovenoso de tolerância à glicose, com dosagem da glicose e insulina plasmáticas, na $32^{\text {a }}$ semana de 917 gestantes não-diabéticas e com feto único. Observaram que variações dentro da faixa de normalidade do metabolismo da glicose materno tinham influências sobre o crescimento e o desenvolvimento fetais. Os efeitos dos níveis glicêmicos maternos sobre o crescimento fetal foram explicados por Pedersen (12).

No Serviço de Obstetrícia do Hospital das Clínicas da Faculdade de Medicina de Botucatu (HCFMB), além do diagnóstico de DMG é também feito o de hiperglicemia diária. Para este, a gestante tem o teste oral de tolerância à glicose (TOTG) normal, mas pelo menos um valor glicêmico alterado: de jejum ou pós-prandial, durante o período diurno de um dia habitual. Rudge e cols. (13) observaram que essas gestantes tiveram com maior freqüência filhos GIG $(53,8 \%)$ que aquelas com DMG (apenas o TOTG alterado) (28,6\%).

Como no Brasil, também é preocupante, em termos de saúde pública, a prevalência crescente de obesidade e suas morbidades associadas na infância e na adolescência, nosso objetivo foi observar se diferentes graus de hiperglicemia durante a gestação: normoglicemia ao perfil glicêmico e TOTG (grupo-controle); apenas o perfil glicêmico alterado (grupo hiperglicemia diária); TOTG alterado e geralmente, também, o perfil glicêmico alterado (grupo DMG) determinam diferentes freqüências de obesidade, DMT2, hipertensão arterial e dislipidemia na infância ou adolescência dos filhos.

\section{MÉTODOS}

Participaram do estudo os filhos de gestantes que foram acompanhadas pelo Serviço de Obstetrícia do HCFMB, no período de 1988 a 1999, e que participaram de estudos prévios. Das 107 mães e 127 filhos pretendidos, foram localizadas e aceitaram participar deste 
estudo 57 mães $(53,3 \%)$ e 73 filhos (57,5\%). As mães e os filhos que não participaram não diferiam dos participantes quanto às variáveis analisadas. Os 73 filhos foram subdivididos em três grupos, segundo a tolerância à glicose materna durante a gestação: 27 no grupo 1 (G1) ou controle, 23 no grupo 2 (G2) ou hiperglicemia diária, 23 no grupo 3 (G3) ou DMG.

O projeto foi previamente aprovado pelo Comitê de Ética em Pesquisa de nossa instituição, e para cada filho tinha-se o consentimento livre e por escrito da mãe.

Cada filho era submetido à avaliação clínica, dando-se atenção especial às medidas antropométricas e da pressão arterial. Tendo observado jejum noturno de 8 a 12 horas, colhia-se uma amostra de sangue venoso periférico para dosagem da glicose, colesterol total e frações e triglicerídeos. Com sua mãe, respondia a um questionário amplo, envolvendo: história da gestaçãoalvo; dados neonatais; hábitos alimentares; grau de escolaridade; estilo de vida; doenças pregressas, aspectos socioeconômicos e história familiar de doenças crônicas mais freqüentes (primeira e segunda gerações). Os dados referentes aos períodos gestacional e neonatal foram complementados por análise dos prontuários médicos.

A glicemia e o perfil lipídico foram dosados pelo método da química seca (Aparelho Vitros $750^{\circledR}$, Johnson \& Johnson, Estados Unidos). Os valores de normalidade foram os preconizados pela Sociedade Americana de Diabetes, 2006 (14) e pela III Diretrizes Brasileiras sobre Dislipidemias, 2001 (15), respectivamente.

A glicemia média diária materna foi calculada dos valores glicêmicos medidos a cada duas horas, durante o período diurno de um dia habitual, duas vezes, durante o terceiro trimestre da gestação.

$\mathrm{O}$ índice de massa corporal (IMC) foi calculado como a razão entre peso $(\mathrm{kg})$ e o quadrado da altura $\left(\mathrm{m}^{2}\right)$ ou a altura $(\mathrm{m})$ para os filhos com $\geq 10$ ou $<10$ anos de idade, respectivamente. Segundo seu valor, foram classificados como eutróficos se entre os percentis $5^{\circ}$ e $85^{\circ}$, como sobrepeso se entre os percentis $85^{\circ}$ e $95^{\circ}$ e como obesos se em percentis acima do $95^{\circ}$. Em todas as faixas etárias e nas três classes de peso relativo à altura há distinção entre os sexos (WHO, 1995) (16).

A circunferência abdominal foi considerada aumentada se igual ou superior a $87 \mathrm{~cm}$ para ambos os $\operatorname{sexos}(17)$.

A prega cutânea tricipital foi classificada de acordo com sexo, idade e raça, sendo considerada adequada se entre os percentis $5^{\circ}$ e $95^{\circ}(18)$.
Os valores pressóricos foram obtidos e considerados normais ou elevados para aqueles com idade até 17 anos e 11 meses, segundo sexo, idade e percentil de estatura, pelos critérios estabelecidos pelo III Consenso Brasileiro de Hipertensão Arterial, 1999 (19). Quando necessário, fez-se a correção das medidas pela circunferência do braço.

A adequação quanto à ingestão familiar ou individual dos vários componentes alimentares foi considerada seguindo os critérios do programa Virtual Nutri ${ }^{\circledR}$ (20), com utilização do recordatório alimentar habitual e a freqüência alimentar.

A atividade física foi classificada de leve, se praticada até três vezes por semana e por 30 minutos em cada vez, a intensa, se diariamente e com duração maior que 30 minutos por dia (21).

\section{Análise Estatística}

$\mathrm{Na}$ comparação entre os grupos quanto às variáveis quantitativas, utilizou-se a técnica da análise de variância para o modelo com um fator paramétrico quando a variável apresentava aderência à distribuição normal de probabilidade e não-paramétrico, na falta desta (22). Quanto às variáveis qualitativas, foram analisadas pelo teste de Goodman para contrastes entre e dentro de populações multinomiais (23). Foram utilizadas letras minúsculas para indicar os resultados das comparações entre grupos, fixada a categoria de resposta, e letras maiúsculas nas comparações das categorias de respostas dentro do grupo. Para a interpretação das letras, devese proceder da seguinte maneira: (a) duas proporções seguidas de pelo menos uma letra minúscula igual não diferem quanto aos respectivos grupos, na categoria da resposta em consideração; (b) duas proporções seguidas de pelo menos uma letra maiúscula igual não diferem quanto às respectivas categorias de resposta dentro do grupo em consideração.

Os resultados são expressos em média \pm desvio-padrão e em porcentagem. Considerou-se significativa uma diferença entre os grupos se $\mathrm{p} \leq 0,05$.

\section{RESULTADOS}

\section{Mães}

As mães dos três grupos, durante a gestação-alvo, não diferiram quanto à idade $(\mathrm{Gl}: 31 \pm 7, \mathrm{G} 2: 33 \pm 10, \mathrm{G} 3$ : $32 \pm 6$ anos; $p>0,05)$, IMC pré-gestação (Gl: $23 \pm 3$, 
G2: $\left.24 \pm 3, \mathrm{G} 3: 25 \pm 3 \mathrm{~kg} / \mathrm{m}^{2} ; \mathrm{p}>0,05\right)$ e ganho ponderal durante a gestação (Gl: $12 \pm 4, \mathrm{G} 2: 11 \pm 5$; G3: $13 \pm 5 \mathrm{~kg} ; \mathrm{p}>0,05)$. A glicemia média de jejum durante a gestação foi semelhante entre G1 e G2 e significativamente mais elevada em G3 (Figura 1). A glicemia média diária na gestação foi significativamente diferente entre os três grupos e com valores crescentes de Gl a G3 (Figura 2). Conseqüentemente, o tratamento com insulina, associado às orientações alimentares, foi necessário para G2 e G3 compreendendo 14,3\% e $30,8 \%$ das gestações, respectivamente $(\mathrm{G} 3>\mathrm{Gl}$; $\mathrm{p}<$ $0,05)$. Também, as mães dos três grupos foram semelhantes quanto à maioria possuir até o $2^{\circ}$ grau de escolaridade, $81 \%$ não terem apresentado hipertensão arterial, $73 \%$ a $81 \%$ não terem fumado durante a gestação e $54 \%$ a $62 \%$ dos partos terem sido via alta.

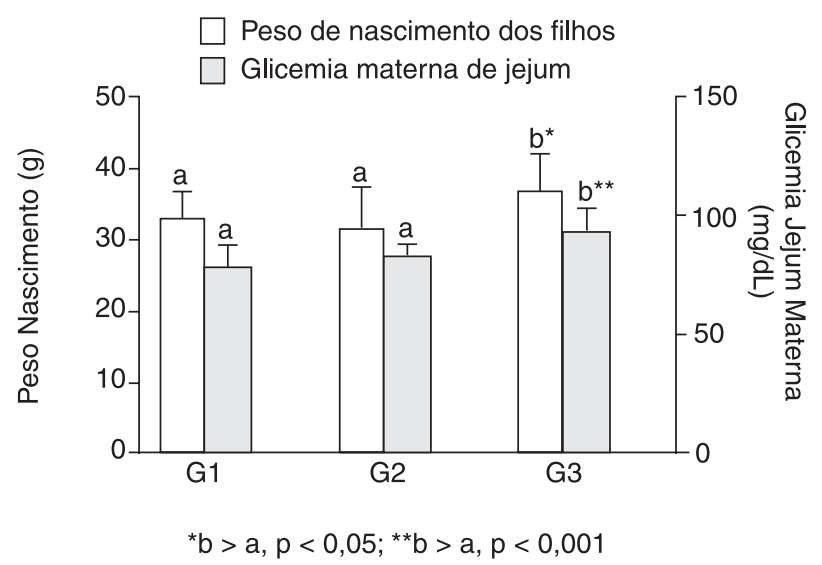

Figura 1. Peso de nascimento dos filhos e glicemia de jejum materna, durante a gestação, dos três grupos

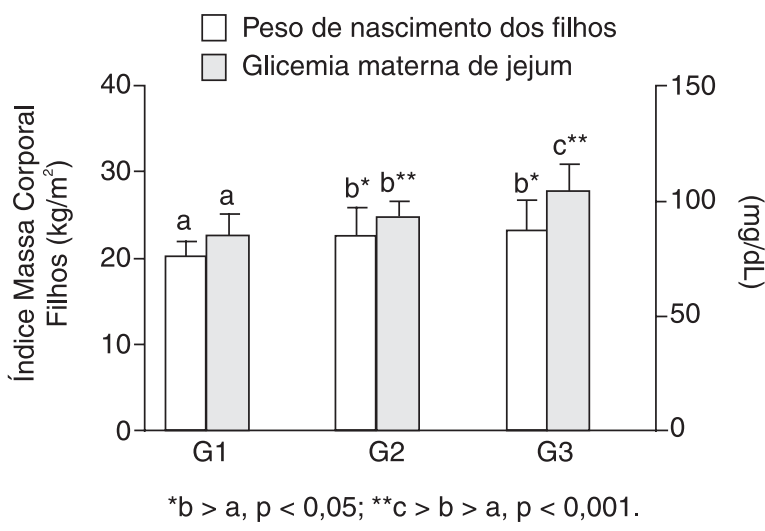

Figura 2. Índice de massa corporal atual dos filhos e glicemia materna diária durante a gestação dos três grupos

\section{Filhos}

A) Período Neonatal

Ao nascimento, os recém-nascidos dos três grupos não diferiram quanto à idade gestacional (Gl: $39 \pm$ 2, G2: $39 \pm 2, \mathrm{G} 3: 38 \pm 2$ sem.; p > 0,05), cerca de $90 \%$ deles a termo. Nos três grupos houve equilíbrio entre os sexos. A maioria nasceu bem, com Apgar do $1^{\circ}$ minuto de 9 e do $10^{\circ}$ minuto de 10 , não havendo diferença entre os grupos. Os recémnascidos dos três grupos não diferiram quanto à estatura (G1: $49 \pm 3, \mathrm{G} 2: 49 \pm 2, \mathrm{G} 3: 50 \pm 2 \mathrm{~cm}$; $\mathrm{p}>0,05)$, todavia, os do G3 apresentaram peso significativamente mais elevado que os dos outros dois grupos, que foram semelhantes entre si (Figura 2 ). Segundo a idade gestacional, o peso de nascimento foi adequado em $57 \%$ a $77 \%$ dos recém-nascidos, não havendo diferença entre os grupos (Tabela 1 ). A duração do aleitamento materno e a idade em que ocorreu a introdução dos alimentos foi de 4 a 6 meses, não havendo diferença entre os grupos. Todos tiveram desenvolvimento neuropsicomotor adequado, exceto duas crianças (G1 e G2) que apresentaram retardo em virtude de meningite e paralisia cerebral, respectivamente.

B) Infância e adolescência

Os participantes do G3 eram mais jovens que os do Gl e do G2, que tinham idade semelhante (Tabela 2). Os filhos G2 e G3 apresentaram IMC significativamente mais elevado que o dos Gl (Figura 2),

Tabela 1. Distribuição da freqüência das classes de peso de nascimento relativo à idade gestacional dos filhos, segundo o grupo

\begin{tabular}{clccc}
\hline \multirow{3}{*}{ Grupo } & \multicolumn{4}{l}{ Classes, peso e nascimento, } \\
& segundo idade gestacional & Total \\
& AIG $^{1}$ & PIG $^{2}$ & GIG $^{3}$ & \\
$G 1$ & $0,769^{a B}$ & $0,192^{a A}$ & $0,038^{a A}$ & 27 \\
$G 2$ & $0,571^{a B}$ & $0,381^{a B}$ & $0,048^{a A}$ & 23 \\
$G 3$ & $0,692^{a B}$ & $0,115^{a A}$ & $0,192^{a A}$ & 23 \\
\hline
\end{tabular}

${ }^{1} \mathrm{AIG}=$ adequado para $\mathrm{a}$ idade gestacional; ${ }^{2} \mathrm{PIG}=$ pequeno para a idade gestacional; ${ }^{3} \mathrm{GlG}=$ grande para $a$ idade gestacional. Duas proporções seguidas de pelo menos uma letra minúscula igual não diferem quanto aos respectivos grupos, na categoria de resposta em consideração; duas proporções seguidas de pelo menos uma letra maiúscula igual não diferem quanto às respectivas categorias de resposta, dentro do grupo em consideração. 
expressando o peso atual com porcentagem do peso ideal, segundo idade e sexo, apenas no G3 ocorreu obesidade $(4,3 \%)$ e $52,2 \%$ eram sobrepesos, freqüência esta significativamente mais elevada que a de G1 (14,8\%) (Tabela 3). Em G2 houve $34,8 \%$ de sobrepeso, freqüência intermediária, mas não significativamente diferente da de Gl e de G3 (Tabela 2). Os três grupos não diferiram quanto à distribuição corporal de gordura (Tabela 2 ), às proporções corporais e ao estadiamento puberal, sendo as medidas adequadas para idade e sexo. Os filhos dos três grupos encontravam-se normotensos e não diferentes entre si (Tabela 2 ).
Todos tinham grau de escolaridade adequado para a idade, exceto a criança que teve paralisia cerebral. Negavam passado mórbido importante. Nenhum era tabagista e $76 \%$ a $92 \%$ não ingeriam bebidas alcoólicas. Todos pertenciam a famílias com renda per capita mediana de um salário mínimo e dispunham de moradia com as condições básicas de saneamento. Os três grupos foram semelhantes quanto à história familiar, sendo que $70 \%$ a $90 \%$ referiam familiares com DMT2, hipertensão arterial e, cerca de $50 \%$, com obesidade ou dislipidemia.

Os participantes dos três grupos relataram hábitos alimentares semelhantes. Referiam ingestão calórica diá-

Tabela 2. Características demográficas atuais dos filhos, segundo o grupo.

\begin{tabular}{|c|c|c|c|}
\hline \multirow[b]{2}{*}{ Variável } & \multicolumn{3}{|c|}{ Grupo (n) } \\
\hline & G1 (27) & G2 (23) & G3 (23) \\
\hline Idade (anos) & $15 \pm 5^{b}$ & $16 \pm 3^{b}$ & $12 \pm 5^{a}$ \\
\hline Peso (kg) & $50 \pm 13$ & $59 \pm 15$ & $50 \pm 22$ \\
\hline Altura $(\mathrm{cm})$ & $156 \pm 17$ & $161 \pm 13$ & $148 \pm 24$ \\
\hline $\mathrm{IMC}^{1}\left(\mathrm{~kg} / \mathrm{m}^{2}\right)$ & $20,3 \pm 1,7^{b}$ & $22,5 \pm 3,4^{b}$ & $23,1 \pm 3,6^{b}$ \\
\hline $\mathrm{PCT}^{2}(\mathrm{~mm})$ & $13,8 \pm 5,7$ & $14,9 \pm 7,3$ & $15,7 \pm 5,8$ \\
\hline $\mathrm{CA}^{3}(\mathrm{~cm})$ & $66,2 \pm 6,1$ & $71,4 \pm 9,3$ & $70,4 \pm 12,1$ \\
\hline $\mathrm{PA}^{4}$ sistólica $(\mathrm{mmHg})$ & $101 \pm 11$ & $107 \pm 14$ & $102 \pm 13$ \\
\hline $\mathrm{PA}^{4}$ diastólica $(\mathrm{mmHg})$ & $68 \pm 10$ & $71 \pm 7$ & $68 \pm 9$ \\
\hline $\mathrm{FP}^{5}(\mathrm{bpm})$ & $68 \pm 13$ & $69 \pm 14$ & $70 \pm 15$ \\
\hline Glicose sérica (mg/dL) & $90 \pm 7$ & $91 \pm 6$ & $93 \pm 7$ \\
\hline Colesterol total sérico (mg/dL) & $156 \pm 30$ & $144 \pm 28$ & $156 \pm 30$ \\
\hline LDL-C sérica (mg/dL) & $87 \pm 25$ & $77 \pm 27$ & $86 \pm 23$ \\
\hline HDL-C sérica (mg/dL) & $51 \pm 13$ & $49 \pm 16$ & $53 \pm 15$ \\
\hline Triglicérides séricos (mg/dL) & $94 \pm 42$ & $84 \pm 21$ & $84 \pm 33$ \\
\hline
\end{tabular}

${ }^{1} \mathrm{IMC}=$ índice de massa corporal; ${ }^{2} \mathrm{PCT}=$ prega cutânea tricipital; ${ }^{3} \mathrm{CA}=$ circunferência abdominal; ${ }^{4} \mathrm{PA}=$ pressão arterial; ${ }^{5} \mathrm{FP}=$ freqüência de pulso; $b>a, p<0,05$.

Tabela 3. Distribuição da classificação dos filhos em relação ao peso atual, como porcentagem do peso ideal (PI), considerando-se sexo e idade, segundo o grupo.

\begin{tabular}{lcccc}
\hline & & Peso atual como \% PI & \\
Grupo & Baixo & Moderado & Alto & Total \\
G1 & $0,852^{\mathrm{bB}}$ & $0,148^{\mathrm{aA}}$ & $0,000^{\mathrm{aA}}$ & 27 \\
G2 & $0,652^{\mathrm{abB}}$ & $0,348^{\mathrm{abB}}$ & $0,000^{\mathrm{aA}}$ & 23 \\
G3 & $0,435^{\mathrm{aB}}$ & $0,522^{\mathrm{bB}}$ & $0,043^{\mathrm{aA}}$ & 23 \\
\hline
\end{tabular}

Duas proporções seguidas de pelo menos uma letra minúscula igual não diferem quanto aos respectivos grupos, na categoria de resposta em consideração; duas proporções seguidas de pelo menos uma letra maiúscula igual não diferem quanto às respectivas categorias de resposta, dentro do grupo em consideração. 
ria média de 2.000 a $2.400 \mathrm{kcal}$, que foi considerada excessiva em $57 \%$ a $65 \%$ dos filhos. O consumo familiar de açúcar e óleo foi semelhante nos três grupos e considerado acima do recomendado. Também o consumo individual de doces, frituras e refrigerantes foi semelhante nos três grupos e predominantemente elevado. $\mathrm{O}$ consumo individual de fibras foi igualmente distribuído nas três categorias quantitativas (baixo, moderado, elevado) nos três grupos.

Nos três grupos, houve predomínio de grau moderado de atividade física.

Os filhos dos três grupos não diferiram quanto aos valores basais séricos da glicose e do perfil lipídico, que estavam na faixa de normalidade para idade e sexo (Tabela 2).

\section{DISCUSSÃO}

As mães dos três grupos durante a gestação diferiram apenas quanto aos níveis glicêmicos que foram crescentes do G1 ao G3. Os filhos tinham condições socioculturais e hábitos de vida semelhantes e próprios das populações ocidentais. Pudemos, assim, observar as conseqüências dos diferentes graus de hiperglicemia materna nos filhos durante o início da vida (período neonatal) e posteriormente (na infância e na adolescência).

A hiperglicemia maior (de jejum e diária) durante a gestação do G3 acompanhou-se de peso de nascimento e IMC mais elevados e maior ocorrência de sobrepeso e obesidade na infância ou na adolescência. A hiperglicemia materna intermediária (apenas a diária) do G2 acompanhou-se de aumento do IMC e da freqüência de sobrepeso na infância ou na adolescência em valores também intermediários entre os de Gl e G3.

O efeito da hiperglicemia durante a gestação no peso de nascimento está de acordo com a hipótese de Pedersen (12). Ela estabelece que a hiperglicemia materna determina hiperglicemia e hiperinsulinemia no feto, que causam sua macrossomia. Em índios Pima (5) e em grupo populacional italiano (24) também os filhos de mães com DMG tiveram maior peso de nascimento que os de mães com tolerância à glicose normal. Nos primeiros, ficou demonstrado o papel maior da hiperglicemia durante a vida intra-uterina em relação a fatores hereditários sobre o peso de nascimento, que foi significativamente mais elevado nos filhos de mães diabéticas na gestação comparativamente àqueles de mães que posteriormente à gestação se tornaram diabéticas e de não- diabéticas (5). Diferentemente dos resultados de Rudge e cols. (13), os recém-nascidos G2 tiveram peso semelhante ao dos Gl e menor que o dos G3. Nos dois estudos, o grupo populacional foi semelhante, mas o número de participantes neste foi menor e talvez o grau de controle glicêmico durante a gestação tenha sido melhor, com glicemia média de jejum e diária de 83 e $93 \mathrm{mg} / \mathrm{dL}$, respectivamente.

A hiperglicemia durante a vida intra-uterina ter se acompanhado de aumento do IMC e de freqüência maior de sobrepeso/obesidade na infância e na adolescência está de acordo com a hipótese de Freinkel (6). Em diferentes grupos populacionais foi demonstrada associação positiva entre a hiperglicemia materna na gestação e a ocorrência de obesidade na infância da prole (3-5,8,22). Nos índios Pima, Lindsay e cols. (5) demonstraram que o estado de tolerância à glicose materno, durante a gestação, sobrepõe-se aos fatores hereditários na determinação de obesidade no futuro dos filhos. O principal fator para essa associação seria a hiperfunção prematura das células $\beta$, observada pelos níveis elevados de insulina no líquido amniótico ou do peptídeo C no sangue do cordão umbilical (4), ou seriam alterações hormonais e de substratos no feto causando distúrbios da regulação de centros nervosos centrais no controle do metabolismo e do peso (3).

O aumento do IMC nos adolescentes do G3 pode ter recebido a influência do peso de nascimento que foi também mais elevado, como observado em estudo anterior com filhos de mães que tiveram DMG (8).

A elevação dos níveis glicêmicos observados nas mães G2 e principalmente nas G3 durante a gestação em relação aos das Gl não alterou a glicemia de jejum dos filhos na adolescência. Mesmo tendo havido aumento do IMC dos filhos G2 e G3 e do peso de nascimento dos G3. Contrariamente, estudos com grupos populacionais com diferentes graus de risco para $\mathrm{O}$ DMT2, como: índios Pima $(5,26)$, canadenses (27), alemães (3) e norte-americanos de Chicago (4), demonstraram por meio do TOTG que a hiperglicemia materna determina maior ocorrência de diabetes na prole. Mesmo em mães não-diabéticas durante a gestação, estudo amplo com índios Pima mostrou que variação de $23 \mathrm{mg} / 100 \mathrm{~mL}$ da glicemia materna determina aumento de $56 \mathrm{~g}$ no peso de nascimento, aumento do peso na infância e risco de 1,6 vez para o desenvolvimento de DMT2 na adolescência e na vida adulta (26). A diferença entre os resultados provavelmente se deve à glicemia de jejum se alterar posteriormente à elevação da 
glicemia pós-prandial ou sua equivalente, a glicemia de duas horas após ingestão de $75 \mathrm{~g}$ de glicose $(27,28)$. Colaborando com essa opinião, temos o estudo nacional norte-americano (Third Health Nutrition Examination Survey, 1988-1994, NHANES III), no qual em 2.430 jovens entre 12 e 19 anos de idade observou-se que dos componentes da síndrome metabólica, a menos freqüente foi a hiperglicemia de jejum, que ocorreu em 1,5\% dos participantes (1).

Estudos prévios com grupos populacionais diversos mostraram uma curva em U quando se relacionava o peso de nascimento e a ocorrência futura de DMT2, em geral diagnosticada por glicemia pós-prandial ou de duas horas após uma carga oral de glicose $(7,29)$. O mecanismo envolvido seria o desenvolvimento de obesidade e conseqüentemente de resistência à insulina $(9,29)$. A ausência dessa relação em nosso estudo se deve a dosagem exclusiva da glicemia de jejum e ao número de participantes relativamente pequeno.

Nos adolescentes G3 também não observamos os outros componentes da síndrome metabólica, como hipertensão arterial e dislipidemia. Nos jovens envolvidos no NHANES III, a dislipidemia foi o componente da síndrome metabólica de maior freqüência, tendo ocorrido em $23 \%$ dos jovens, e a hipertensão arterial teve ocorrência intermediária, de 4,9\% (1). A diferença de resultados ocorre por este estudo ser populacional e o nosso, amostral.

Quanto à presença da síndrome metabólica em filhos de mães diabéticas durante a gestação, se por um lado nossos resultados diferem dos de Boney e cols. (30), que verificaram em crianças caucasianas, residentes na Rhode Island, com 6 a 11 anos de idade a elevada freqüência de $50 \%$ naquelas cujas mães tiveram DMG e que nasceram GIG, por outro lado, são semelhantes aos de Silva e cols. (31). Estes autores não observaram nenhum dos componentes da síndrome metabólica em 30 jovens brasileiros com sobrepeso e parentes em primeiro grau com DMT2.

Concluindo, para diminuir a ocorrência de sobrepeso e mesmo obesidade na adolescência e certamente também na vida adulta, é necessário detectar o mais precocemente possível o DMG e nessas gestantes, procurar um ótimo controle das glicemias de jejum $(<90 \mathrm{mg} /$ $\mathrm{dL})$ e pós-prandiais $(<130 \mathrm{mg} / \mathrm{dL})$. Os filhos dessas mães precisam ser acompanhados e educados para a aquisição de hábitos de vida saudáveis o mais precocemente possível. Sugerimos que as gestantes, a partir da $24^{\mathrm{a}}, 28^{\mathrm{a}}$ semana, sem diagnóstico de DMG, também sejam avalia- das quanto às glicemias de jejum e pós-prandiais e, quando elevadas, tratadas e acompanhadas de forma semelhante às com DMG. Seus filhos também devem ser acompanhados como o preconizado para aqueles de mães que tiveram DMG.

Para confirmação dos resultados, seria interessante a ampliação do número de participantes e o acompanhamento longitudinal destes.

\section{AGRADECIMENTOS}

O projeto recebeu Auxílio à Pesquisa da Fundação para o Desenvolvimento da Universidade Estadual Paulista (Fundunesp) - Processo $\mathrm{n}^{\circ}$ 00548/04-DFP.

\section{REFERÊNCIAS}

1. Cook $S$, Weitzman $M$, Auinger $P$, Nguyen $M$, Dietz WH. Prevalence of a metabolic syndrome phenotype in adolescents. Arch Pediatr Adolesc Med. 2003;157:821-7.

2. Veiga GV, Cunha AS, Sichieri R. Trends in overweight among adolescents living in the poorest and richest regions of Brazil. Am J Public Health. 2004;94:1544-8.

3. Plagemann A, Harder T, Kohlhoff R, Rohde W, Dörner G. Overweight and obesity in infants of mothers with long-term insulin-dependent diabetes or gestational diabetes. Int J Obes. 1997;21:451-6.

4. Silverman BL, Rizzo TA, Cho NH, Metzger BE. Long-term effects of the intrauterine environment: the Northwestern University Diabetes in Pregnancy Center. Diabetes Care. 1998;21(2 suppl):142B-149B.

5. Lindsay RS, Hanson RL, Bennett PH, Knowler WC. Secular trends in birth weight, BMI, and diabetes in the offspring of diabetic mothers. Diabetes Care. 2000;23:1249-54.

6. Freinkel N. Banting Lecture 1980: of pregnancy and progeny. Diabetes. 1980; 29:1023-35.

7. Schaefer-Graf UM, Pawliczak J, Passow D, Hartmann R, Rossi $\mathrm{R}$, Bührer $\mathrm{C}$, et al. Birth weight and parental BMI predict overweight in children from mothers with gestational diabetes. Diabetes Care. 2005;28:1745-50.

8. Vohr BR, McGarvey ST, Tucker R. Effects of maternal gestational diabetes on offspring adiposity at 4-7 years of age. Diabetes Care. 1999;22:1284-91.

9. Eriksson JG, Forsen TJ, Osmond C, Barker DJP. Pathways of infant and childhood growth that lead to type 2 diabetes. Diabetes Care. 2003;26:3006-10.

10. Tallarigo L, Giampietro O, Penno G, Miccoli R, Gregori G, Navalesi R. Relation of glucose tolerance to complications of preg nancy in nondiabetic women. N Engl J Med. 1986;315:989-92.

11. Farmer G, Russell G, Hamilton-Nicol DR, Ogenbede HO, Ross JS, Pearson DWM, et al. The influence of maternal glucose metabolismo on fetal growth, development and morbidity in 917 singleton pregnancies in nondiabetic women. Diabetologia. 1988;31:134-41.

12. Pedersen J. Weight and length at birth of infants of diabetic mothers. Acta Endocrinol. 1954:16:330-42. 
13. Rudge MVC, Peraçoli JC, Berezowski AT, Calderon IMP, Brasil MAM. The oral glucose tolerance test is a poor predictor of hyperglycemia during pregnancy. Braz $J$ Med Biol Res. 1990;23:1079-89.

14. American Diabetes Association. Diagnosis and classification of diabetes mellitus. Diabetes Care. 2006;29(suppl 1):S43-S48.

15. III Diretrizes Brasileiras sobre Dislipidemias e Diretriz de Prevenção da Aterosclerose do Departamento de Aterosclerose da Sociedade Brasileira de Cardiologia. Arq Bras Cardiol. 2001;77(supl III):1-48.

16. World Health Organization - WHO. Physical status: the use and interpretation of anthropometry. WHO Technical Report Series n. 854. Geneva: WHO; 1995.

17. Li C, Ford ES, Mokadad AH, Cook S. Recent trends in waist circumference and waist-height ratio among US children and adolescents. Pediatrics. 2006;118:e1390-8.

18. Cronk CE, Roche AF. Race and sex-specific reference data for triceps and subscapular skinfolds and weight/statura. Am J Clin Nutr. 1982;35:347-54.

19. III Consenso Brasileiro de Hipertensão Arterial. Rev Bras Cardiol. 1999;1:96-132.

20. Philippi SI, Szarfarc SC, Latterza AR. Virtual Nutri (Programa de computador). Versão 1,0 para Windows. São Paulo: Departamento de Nutrição da Faculdade de Saúde Pública da USP; 1996.

21. Katch FI, McArdle WD. Nutrition, weight control and exercise. 3red. Philadelphia: Lea \& Febiger, 1988.

22. Zar JH. Biostatistical analysis. 4ed. New Jersey: Prentice-Hall, 1999.

23. Goodman LA. Simultaneous confidence intervals for contrasts among multinomial populations. Ann Math Stat. 1964;716-25.

24. Breschi MC, Seghieri G, Bartolomei G, Gironi A, Baldi S, Ferrannini $\mathrm{E}$. Relation of birthweight to maternal plasma glucose and insulin concentrations during normal pregnancy. Diabetologia. 1993;36:1315-21.
25. Gerlini G, Arachi S, Gori MG, Gloria F, Bonci E, Pachi A, et al. Developmental aspects of the offspring of diabetic mothers. Acta Endocrinol. 1986;(Suppl) 277:150-5.

26. Franks PW, Looker HC, Kobes S, Touger L, Tataranni PA, Hanson $\mathrm{RL}$, et al. Gestational glucose tolerance and risk of type 2 diabetes in young Pima Indian offspring. Diabetes. 2006;55:460-5.

27. Malcolm JC, Lawson ML, Gaboury I, Lough G, Keely E. Glucose tolerance of offspring of mother with gestational diabetes mellitus in a low-risk population. Diabetic Med. 2006;23:565-70.

28. Le Roith D. $\beta$-cell dysfunction and insulin resistance in type 2 diabetes: Role of metabolic and genetic abnormalities. Am J Med. 2002;113(6A):3S-11S.

29. Dabelea D, Pettitt DJ, Hanson RL, Imperatore G, Bennett PH, Knowler WC. Birth weight, type 2 diabetes, and insulin resistance in Pima Indian children and young adults. Diabetes Care. 1999;22:944-50.

30. Boney CM, Verma A, Tucker R, Vohr BR. Metabolic syndrome in childhood: Association with birth weight, maternal obesity, and gestational diabetes mellitus. Pediatrics. 2005;115:e290-6.

31. Silva RCQ, Miranda WL, Chacra AR, Dib SA. Metabolic syndrome and insulin resistance in normal glucose tolerant Brazilian adolescents with family history of type 2 diabetes. Diabetes Care. 2005; 28:716-8.

Endereço para correspondência:

Walkyria de Paula Pimenta

Departamento de Clínica Médica

Faculdade de Medicina de Botucatu, Unesp

18918-670 Botucatu, SP

E-mail: wpimenta@fmb.unesp.br 